\title{
The perceptual classification of speech
}

\author{
PETER W. JUSCZYK \\ Dalhousie University, Halifax, Nova Scotia \\ LINDA B. SMITH \\ Indiana University, Bloomington, Indiana \\ and \\ CHRISTOPHER MURPHY \\ Dalhousie University, Halifax, Nova Scotia
}

\begin{abstract}
By employing new methods of analysis to the physical signal, a number of researchers have provided evidence which suggests that there may be invariant acoustic cues which serve to identify the presence of particular phonetic segments (e.g., Kewley-Port, 1980; Searle, Jacobson, \& Rayment, 1979; Stevens \& Blumstein, 1978). Whereas previous studies have focused upon the existence of invariant properties present in the physical stimulus, the present study examines the existence of any invariant information available in the psychological stimulus. For this purpose, subjects were asked to classify either a series of full-CV syllables ([bi], [be], [bo],

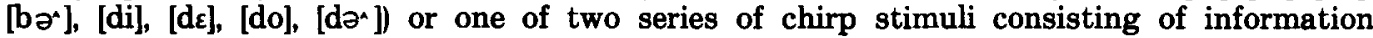
available in the first $30 \mathrm{msec}$ of each syllable. The full-formant chirp stimuli consisted of the first $30 \mathrm{msec}$ of each syllable, whereas the two-formant chirps were composed of the first 30 msec of only the second and third formants. The object of the present study was to determine whether or not there was sufficient information available in either the full- or twoformant chirp series to allow subjects to group the stimuli into two classes corresponding to the identity of the initial consonant of the syllables (i.e., [b], or [d]). A series of classification tasks were used, ranging from a completely free sorting task to a perceptual learning task with experimenter-imposed classifications. The results suggest that there is information available in the full-formant chirps, but not in the two-formant chirps, which allows subjects to group the sounds into classes corresponding to the identity of the initial consonant sounds.
\end{abstract}

A central problem in speech perception is how a listener is able to recognize the presence of a particular phone in all of its possible utterance contexts. A straightforward solution to this problem would be to isolate a particular phone in the speech stream and to identify the property or properties which signal its presence in all contexts. There are several dif-

\footnotetext{
This research was supported by an N.S.E.R.C. grant (A-0282) to the first author and an N.S.F. grant (BNS 78-13019) to the second author. Portions of this research were presented earlier in a paper given at the 99th meeting of the Acoustical Society of America, April 22, 1980, in Atlanta, Georgia. The authors would like to acknowledge the support of a number of persons, including: Diane Kewley-Port for her help in deriving the onset spectra for the stimuli; Joel Katz and the late Frank Restle for their suggestions about the data analysis; Janice Murray for her help in scoring the results; Mike Posner for comments on an earlier version of the manuscript; and especially David Pisoni for his suggestions throughout this research project and for so graciously making available the facilities of the Speech Perception Laboratory at Indiana University. Finally, we would like to thank Harris Savin, who pointed out to one of us some years ago the fundamental problems that exist in determining what constitutes an appropriate nonspeech control. Peter Jusczyk is now at the Department of Psychology, University of Oregon, Eugene, Oregon 97403. Reprint requests should be addressed to him there.
}

ficulties with this approach. First, it is not always possible to isolate and segment phones from the speech stream. Because of coarticulation, information about more than one phone is frequently present over any given interval in time. Thus, attempts to divide a syllable such as [di] into two segments corresponding to [d] and [i] have been unsuccessful (Liberman, Cooper, Shankweiler, \& StuddertKennedy, 1967). As larger sections of the vocalic portion of the syllable are removed, the listener's perception of the remaining portion changes from hearing a shortened [di] to hearing a nonspeech sound. At no point does the listener report perceiving only [d] (but see Kewley-Port, 1980).

Although it is not always possible to isolate individual phonetic segments, one could still ask whether there are invariant properties in the utterance which indicate the presence of a particular phone. Early attempts to isolate such properties were based upon measurements obtained via spectrographic analyses of speech. These analyses indicated that, while some phones appear to exhibit relatively stable properties in different phonetic contexts, such as the characteristics of the noise spectra accompanying [s] and [š] (Harris, 1958), other phones appear to 
undergo considerable context-conditioned variation. For example, studies with two-formant synthetic speech sounds indicate that [d] can be signaled by a rising second-formant transition in some contexts, such as [di], and a falling one in others, such as [du] (Liberman et al., 1967). Not only are invariant properties lacking in the spectrographic representations of [d] across different phonetic contexts, but also the same acoustic property may even cue entirely different phones, depending upon the following vowel context. For example, the same burst of sound is heard as [p] when placed before an [i], but as [k] when before an [a] (Liberman, DeLattre, \& Cooper, 1952; Schatz, 1954). ${ }^{1}$ Findings such as these led some researchers to suppose that at least some phones are without invariant properties in the acoustic signal (Liberman et al., 1967). This belief was propagated by the failure to find such properties in spectrographic analyses (Liberman et al., 1967).

Recently, some investigators have begun to employ other methods to analyze the physical nature of speech. Three efforts which have achieved some success in specifying possible physical invariant cues are those by Kewley-Port (1980), Searle, Jacobson, and Rayment (1979), and Stevens and Blumstein (1978, in press). The methods used by Kewley-Port (1980) and by Searle et al. (1979) are based upon current understanding of the physiology and psychophysics of the auditory system. Searle et al. compute a series of running spectra for speech sounds from which certain features are extracted to determine what phone was uttered. Although encouraging, the recognition accuracy of their model $\mathbf{7 7 \%}$ for detection and classification of stop consonants in initial position) still falls short of that obtained in human speech perception. Using slightly different filtering parameters and a more fine-grained analysis of running spectra, Kewley-Port's (1980) model approached the accuracy of human speech perception. Under test conditions similar to those employed by Searle et al., Kewley-Port's model obtained an overall recognition accuracy score of $\mathbf{8 8 \%}$.

The approach taken by Stevens and Blumstein (1978, in press; Blumstein \& Stevens, 1979) is based upon considerations derived from the acoustic theory of speech production (Fant, 1960). The basic notion behind this approach is that, owing to the acoustic properties of the cavities in the vocal tract, the spectrum sampled over the $10-20$ msec immediately following consonantal release will have distinctive overall characteristics for different places of articulation. The time window that Stevens and Blumstein use in computing the onset spectrum is long enough to include both burst and some formant transition information. However, unlike earlier analyses, which tended to examine each of these cues independently, the cues occur together in an integrated form in the onset spectra. Hence, invariants for speech sounds are presumed to lie in the overall gross shape of the spectrum at onset. As a test of the accuracy of their model, Blumstein and Stevens (1979) examined a large number of $\mathrm{CV}$ and $\mathrm{VC}$ tokens containing both voiced and voiceless stops produced by several speakers. Despite some variability across vowel contexts, the model attained an overall correct identification rate of $85 \%$ for CV syllables. However, recognition accuracy for VC syllables was only $76 \%$. Moreover, a later test of the generality of the model to nasal stops was considerably less successful, since the false alarm rate for the model was as high as $67 \%$ in some instances.

These recent gains in identifying potential acoustic invariants have come about because investigators have employed new ways of describing the physical signal. The goal of these investigators is to discover a description of the physical signal under which physical properties associated with a particular phone remain constant across various contexts. The hope is that if such a description were found, it might correspond to the one used by the perceiver in recognizing speech. However, the best physical description of a set of stimuli need not correspond to the best psychological description (Boring, 1926, 1933; Gibson, 1966; Smith \& Kemler, 1978). A description of the speech signal in terms of some invariant physical properties will provide a successful account of speech perception only insofar as it isolates those properties on which the perceiver operates. Therefore, a converging way of approaching the invariants issue would be to begin with a description of the psychological stimulus. At what point, if any, in the analysis of the speech signal does the listener behave as though there is invariant information common to all instances of a particular phone?

Recent research has begun to address this question (Blumstein \& Stevens, 1980; Kewley-Port, 1980). In a series of studies, Blumstein and Stevens (1980) collected identification data for brief segments excerpted from the initial portions of synthetic consonantvowel stimuli. Subjects labeled these stimuli as [b], [d], or [g], with a high level of accuracy even when the segments were as short as $10-20$ msec. Moreover, in most instances, subjects were also able to correctly label the vowel of the syllable from which the stimulus had been excerpted.

Blumstein and Stevens' (1980) results indicate that there is information available in the onset spectra of CV syllables which the perceiver can use to determine the identity of a particular phone. These results, taken together with their earlier work (Stevens \& Blumstein, 1978), would seem to suggest that the invariant properties derived from their analyses of the physical stimulus are present as well in some form in the psychological stimulus. However, before this 
conclusion can be accepted, several factors must be considered. First of all, since Blumstein and Stevens (1980) always conducted their identification tests separately for each vowel context, it is possible that subjects employed different cues to establish the identity of a particular phone in each context. Certainly, the fact that subjects were quite successful in recognizing the following vowel is consistent with the use of context-dependent cues to establish the identity of the consonant. Second, by instructing their subjects to label the stimuli as [b], [d], or [g], Blumstein and Stevens imposed a constraint on the kinds of perceptual categories that subjects could form. The imposition of such constraints makes it difficult to know whether a categorization according to [b], [d], and [g] represents the most natural psychological grouping of the sounds.

The main objective of the present study was to determine whether brief segments of speech at the onsets of syllables contain information for phones in a form that is usable to the perceiver. While the basic objectives of the two studies are similar, the approach taken in the present study differs in several notable ways from that of Blumstein and Stevens (1980). First, we employed free classification tasks to determine subjects' natural groupings for brief segments of speech. In such tasks, subjects are essentially free to group the stimuli in any way they wish. Second, in instances in which subjects were directed to classify according to phonetic categories, their performance was assessed by comparing it to that obtained when they were instructed to sort according to an arbitrary classification scheme. Inclusion of such a comparison made it possible to test whether a classification according to a phonetic invariant was any more psychologically real for subjects than an arbitrarily chosen grouping. Third, a variety of vowel contexts were present in all classification tasks. Fourth, in addition to utilizing brief segments of synthetic speech with a full complement of formants, the present study also employed both complete syllables and brief two-formant patterns from which the first formant had been deleted. Classification of the latter stimuli was examined because it has been claimed that the deleted first-formant information is redundant across different places of articulation (e.g., Mattingly, Liberman, Syrdal, \& Halwes, 1971). The effect of deleting such "redundant" material on the perceptual categories of brief segments is of interest-especially since these "chirplike" patterns have been used as nonspeech controls in many experiments (e.g., Mattingly et al., 1971; Morse, 1972). The specific questions addressed in the present study include the following: (1) Are brief segments of speech corresponding to one consonant (e.g., [b]) perceived to be more similar to each other than ones corresponding to different consonants (e.g., [b] and [d])? (2) Might such segments be perceived as falling into categories isomorphic to ones organized by initial phonetic segments? (3) More specifically, if subjects are presented with brief speech segments corresponding to [be, be, bo, bə*] and [di, de, do, də*], will they perceive these segments as belonging to two distinct perceptual categories- " $b$ " and " $d$ "?

\section{EXPERIMENT 1}

The hypothesis that brief segments of speech contain invariant information leading to the recognition of particular phones implies that all segments containing the same invariants are perceived as being similar. This hypothesis does not imply that the listener is necessarily able to label all the segments corresponding to a particular phone (say, [d]) as sounding like "d." The critical question is whether listeners perceive brief segments corresponding to [d] as distinct from those corresponding to [b]. Thus, Experiment 1 consisted of a classification task in which subjects were free to classify the stimuli in any way they chose. Our question was whether subjects easily and naturally group brief segments of speech into categories corresponding to phonetic ones.

Three types of stimuli were employed to address this issue: complete $\mathrm{CV}$ syllables, truncated versions of these syllables containing only the first $30 \mathrm{msec}$ of each formant (hereafter referred to as "fullformant chirps"), and truncated versions of the syllables containing only the first $\mathbf{3 0} \mathrm{msec}$ of the second and third formants (hereafter called "two-formant chirps"). Full CV syllables were included, since it was expected that subjects might zasily sort these stimuli into categories based on the identity of their initial consonants. The full-formant chirps were employed because these segments preserved the onset spectra information which Stevens and Blumstein (in press) have claimed provides the invariant phonetic cues. Finally, two-formant chirps were examined because such patterns have often been used to present listeners with the "same acoustic information" in a nonspeech context that is present in a speech context (e.g., Eimas, 1975; Mattingly et al., 1971; Miyawaki, Strange, Verbrugge, Liberman, Jenkins, \& Fujimura, 1975; Morse, 1972). If the two-formant chirps really do preserve the same acoustic information as the syllables, then one might expect to observe similar sorting patterns for these two types of stimuli.

\section{Method}

Stimuli. The stimuli consisted of eight synthetic speech syllables [bi, be, bo, br, di, de, do, dol plus the two truncated versions of each. All stimuli were prepared on a PDP-11/05 

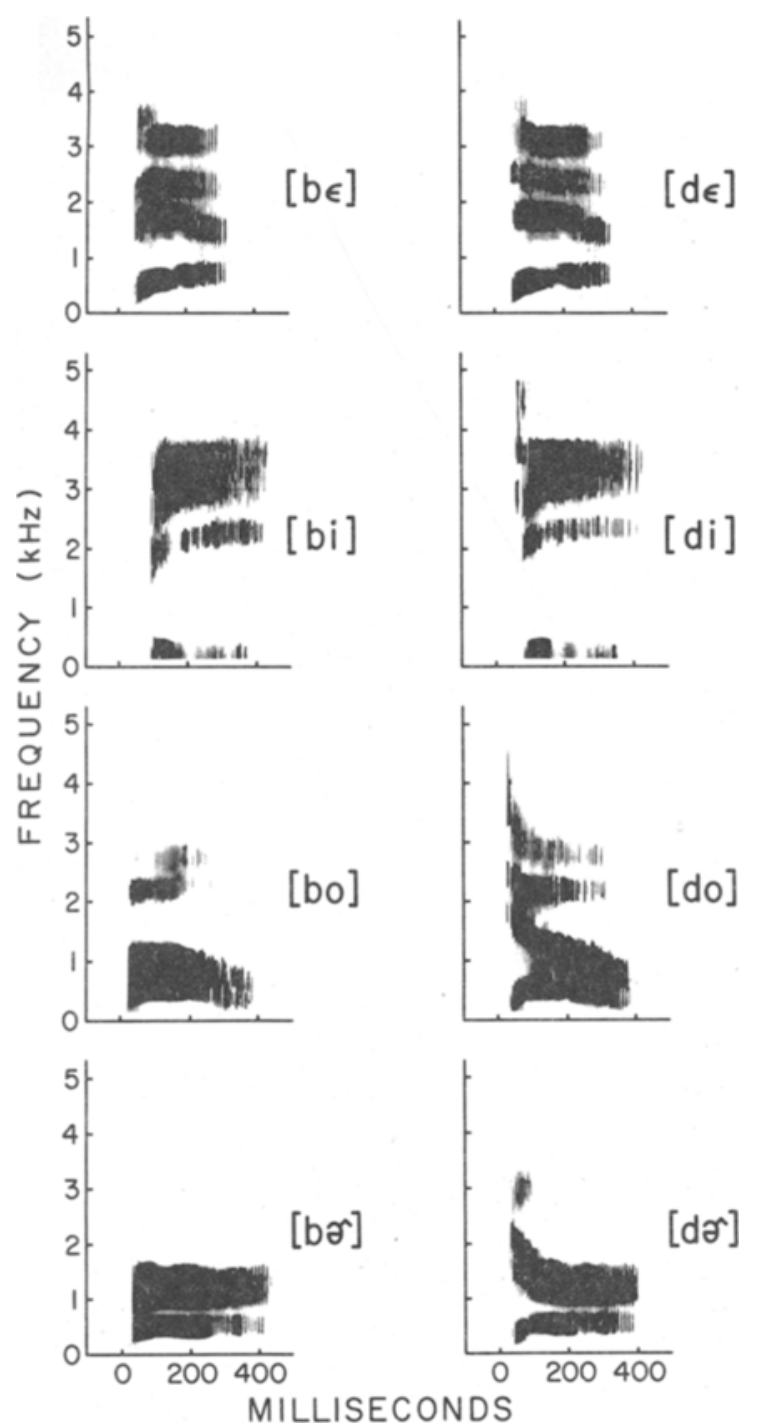

Figure 1. A spectrographic display of the natural $\mathrm{CU}$ syllables which served as models for the synthetic speech tokens used in the present study.

computer in the Speech Perception Laboratory at Indiana University and were generated with the cascade-parallel synthesizer designed by Klatt (1980) and modified by Kewley-Port (Note 1). The eight natural speech tokens spoken by P.W.J. that served as models for constructing the synthetic tokens are displayed in Figure 1. The specific range of vowel contexts included in the syllables was chosen to maximize differences between the relationship of the first, second, and third formants. This measure was adopted so as to provide the strongest possible test of potential invariant cues to the initial consonants.

The syllable stimuli were all generated without release bursts and were equated for overall duration $(295 \mathrm{msec})$ and pitch contour. The latter had an initial value of $121 \mathrm{~Hz}$, rose to a peak of $125 \mathrm{~Hz}$ after $45 \mathrm{msec}$, and then fell linearly to a terminal value of $100 \mathrm{~Hz}^{2}$ Syllables sharing a common vowel (e.g., [bo] and [do]) were equated in all respects except for their second- and third-formant transition values. Table 1 presents the values of the first-, second-, and third-formant values sampled at four points in the duration of each test syllable.

Full-formant chirps for each syllable were produced by truncating the syllable after $30 \mathrm{msec}$, at which point the transitions of the first, second, and third formants were complete. Thus, the relevant formant trajectories are identical to those of the full syllables and are displayed in the first two columns of Table 1 . Moreover, since the full-formant chirps are merely truncated versions of the complete syllables, the onset spectrum for a given full-formant chirp is identical to that of the syllable from which it is derived. The onset spectra for the syllables were analyzed in the same way as Stevens and Blumstein (1978), using the linear prediction method and by preemphasizing the higher frequencies and employing a 26-msec time window (Kewley-Port, Note 2). ${ }^{3}$ The resulting spectra for the stimuli are displayed in Figure 2.

The two-formant chirps were generated by essentially removing the first-, fourth-, and fifth-formant information from the fullformant chirps. However, since the removal of this information can result in a drastic change in the amplitude relations between the second and third formants, measurements of the amplitudes of the transition portions of these formants were made from each syllable using the KLAMP program devised by Kewley-Port. The two-formant chirp patterns were then generated on the parallel branch of the Klatt synthesizer, taking care to maintain the appropriate amplitude relations of the formants throughout the duration of the chirps. Owing to the lack of acoustic energy in the regions of the first, fourth, and fifth formants, the onset spectra for the two-formant chirps differ considerably from those of the syllables and full formants. Figure 3 presents the onset spectra for the two-formant chirps.

Nine test tapes were generated using an audio tape-making program at Indiana University. The stimuli were converted to analogue form in real time via a 12-bit digital-to-analogue converter, low-pass filtered at $4.8 \mathrm{kHz}$, and recorded on a Crown Model 822

Table 1

First, Second, and Third Formants Transitions (0-30 Msec) and Steady State Frequencies (Corresponding to the Following Vowel: 31-295 Msec) in Hertz for the Eight Syllables Sampled at Four Points

\begin{tabular}{|c|c|c|c|c|c|}
\hline & $\begin{array}{l}\text { Formant } \\
\text { Transition }\end{array}$ & $0 \mathrm{Msec}$ & 30 Msec & $200 \mathrm{Msec}$ & $295 \mathrm{Msec}$ \\
\hline /bi/ & $\begin{array}{l}1 \\
2 \\
3\end{array}$ & $\begin{array}{r}200 \\
1500 \\
2200\end{array}$ & $\begin{array}{r}300 \\
2000 \\
2800\end{array}$ & $\begin{array}{r}249 \\
2160 \\
3057\end{array}$ & $\begin{array}{r}220 \\
2250 \\
3200\end{array}$ \\
\hline$/ \mathrm{di} /$ & $\begin{array}{l}1 \\
2 \\
3\end{array}$ & $\begin{array}{r}200 \\
1900 \\
3300\end{array}$ & $\begin{array}{r}300 \\
2000 \\
2800\end{array}$ & $\begin{array}{r}249 \\
2160 \\
3057\end{array}$ & $\begin{array}{r}220 \\
2250 \\
3200\end{array}$ \\
\hline$|\mathrm{b} \epsilon|$ & $\begin{array}{l}1 \\
2 \\
3\end{array}$ & $\begin{array}{r}200 \\
1400 \\
2100\end{array}$ & $\begin{array}{r}550 \\
1850 \\
2500\end{array}$ & $\begin{array}{r}678 \\
1850 \\
2500\end{array}$ & $\begin{array}{r}750 \\
1650 \\
2500\end{array}$ \\
\hline$/ \mathrm{d} \epsilon \mid$ & $\begin{array}{l}1 \\
2 \\
3\end{array}$. & $\begin{array}{r}200 \\
2250 \\
3200\end{array}$ & $\begin{array}{r}550 \\
1850 \\
2500\end{array}$ & $\begin{array}{r}678 \\
1850 \\
2500\end{array}$ & $\begin{array}{r}750 \\
1650 \\
2500\end{array}$ \\
\hline /bo/ & $\begin{array}{l}1 \\
2 \\
3\end{array}$ & $\begin{array}{r}250 \\
650 \\
1700\end{array}$ & $\begin{array}{r}500 \\
1050 \\
2240\end{array}$ & $\begin{array}{r}500 \\
1050 \\
2240\end{array}$ & $\begin{array}{r}350 \\
800 \\
2240\end{array}$ \\
\hline /do/ & $\begin{array}{l}1 \\
2 \\
3\end{array}$ & $\begin{array}{r}250 \\
1800 \\
3000\end{array}$ & $\begin{array}{r}500 \\
1050 \\
2240\end{array}$ & $\begin{array}{r}500 \\
1050 \\
2240\end{array}$ & $\begin{array}{r}350 \\
800 \\
2240\end{array}$ \\
\hline$/ \mathrm{b} x$ & $\begin{array}{l}1 \\
2 \\
3\end{array}$ & $\begin{array}{r}200 \\
800 \\
1100\end{array}$ & $\begin{array}{r}600 \\
1200 \\
1600\end{array}$ & $\begin{array}{r}600 \\
1200 \\
1600\end{array}$ & $\begin{array}{r}600 \\
1200 \\
1600\end{array}$ \\
\hline$/ \mathrm{d} \boldsymbol{r}$ & $\begin{array}{l}1 \\
2 \\
3\end{array}$ & $\begin{array}{r}200 \\
1600 \\
2600\end{array}$ & $\begin{array}{r}600 \\
1200 \\
1600\end{array}$ & $\begin{array}{r}600 \\
1200 \\
1600\end{array}$ & $\begin{array}{r}600 \\
1200 \\
1600\end{array}$ \\
\hline
\end{tabular}

Note-The relationship between the frequencies of all adjacent samples is linear. 

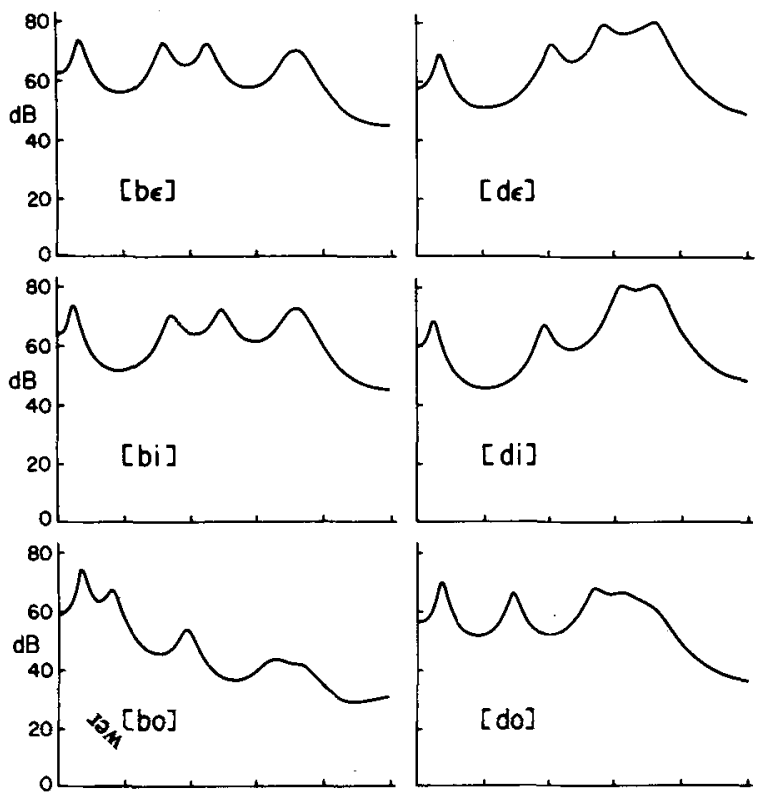

[do]

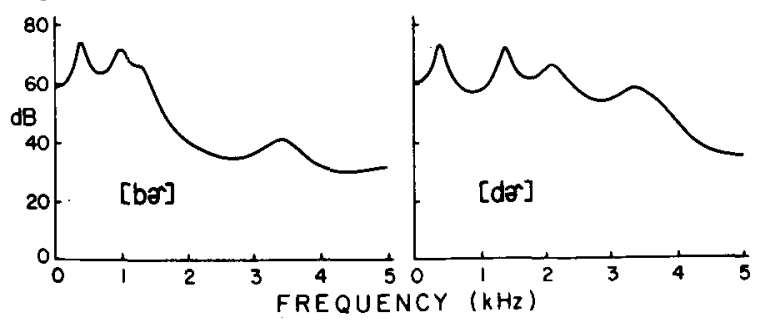

Figure 2. A display of the onset spectra for both the full-syllable and full-formant chirp stimuli.

tape recorder at $71 / 2$ ips. There were three tapes for each of the three stimulus conditions (i.e., full-CV syllables, full-formant chirps, and two-formant chirps). For the syllable condition, each tape included randomized sequences of the eight syllables [bi, bs, bo, bə^, di, de, do, də^]. There were 10 occurrences of each syllable for a total of 80 trials per tape. A 4-sec response interval separated successive syllables on each tape. The tapes for the fullformant and two-formant chirp conditions were prepared in an identical fashion with the appropriate chirps being substituted for syllables. In addition to the test tapes, four tapes were prepared for each stimulus condition for the purpose of familiarizing the subjects with the stimulus sets.

Subjects. The subjects were 15 undergraduates at Dalhousie University, who received course credit for participating in the experiment. All were native speakers of English and reported no history of either speech or hearing disorder.

Procedure. Each subject was tested individually in a small quiet room for one $1 / 2-h$ session. Practice and test tapes were played on a Tandberg TB-3500 X tape recorder equipped with Koss PRO 4AAA headphones. The volume was adjusted with reference to a sound-level meter (General Radio Model 1565-A) so that the stimuli were played at a level of approximately $72 \mathrm{~dB}$ (A) SPL.

An equal number of subjects were assigned randomly to each of the three stimulus conditions. The subjects were instructed that they would be hearing a series of eight sounds and that they would have to sort the stimuli into groups. No explicit rule was provided for the subjects; rather, they were told to "put together the stimuli which sounded the most alike." The subjects were informed that they were free to form as many groups as they

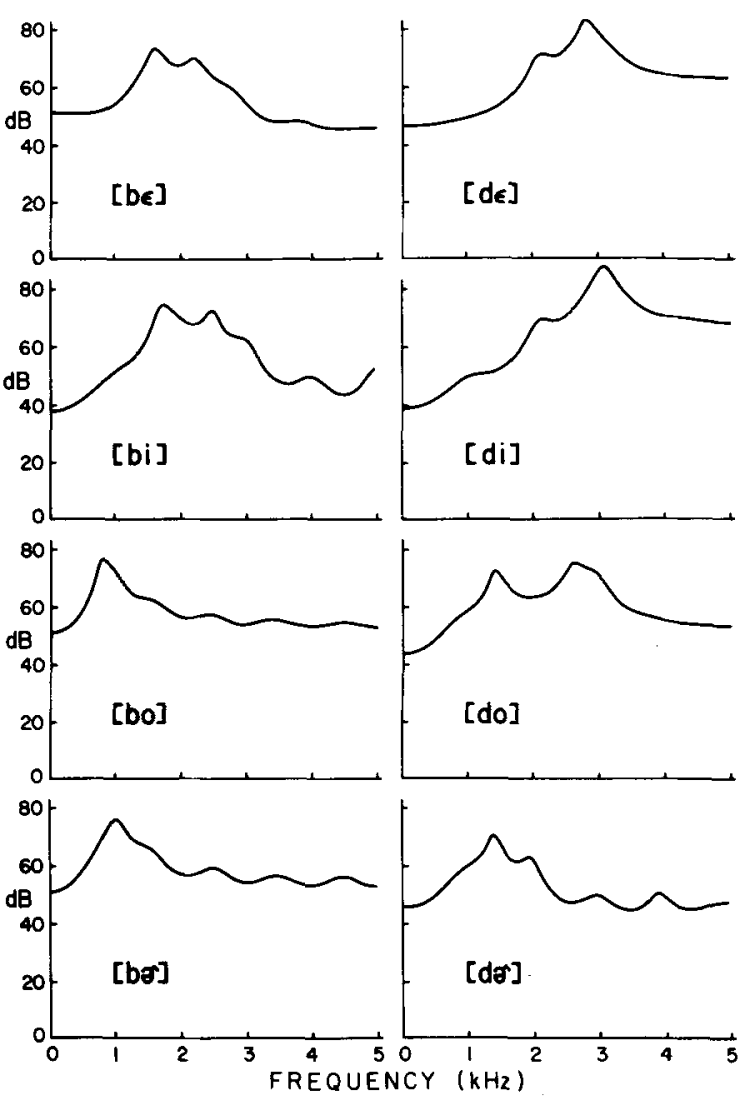

Figure 3. A display of the onset spectra of the two-formant chirp stimuli.

desired. Following these instructions, the subjects heard one of the demonstration tapes containing one occurrence of all eight stimuli so that they might familiarize themselves with the entire set of sounds and form a classification system. Two more demonstration tapes with the eight test items in different random orders were then played, and the subjects were asked to practice assigning the stimuli to groups. The subjects were told to use numbers to designate the different groups and to indicate the assignment of a particular stimulus to a group by writing down the group number in an appropriately marked space on their answer sheet. After the practice trials, the subjects were administered one 80 -item test tape. A short 5 min break ensued, during which subjects were told that they were free to change the way in which they grouped the stimuli, if they wished, on the next block of trials. The demonstration and practice sequences were repeated prior to the administration of the second test tape (with a different random ordering of the stimuli). Before departing, all subjects completed a short questionnaire regarding the criteria and strategies they had employed in grouping the stimuli.

\section{Results and Discussion}

Because subjects were often still familiarizing themselves with the stimulus materials during the initial test series (especially with the chirp stimuli), only the data from the second 80 -item test series were submitted to analysis. The first consideration was whether subjects were consistent in putting a given stimulus in a particular group or whether they changed 
the group to which the stimulus was assigned from trial to trial during the test. Inconsistent classifications of particular sounds might result from difficulties in identifying the stimuli and/or remembering one's groups. To assess the overall consistency of subjects' classification of the stimuli, a consistency measure was devised using the Relative $\mathbf{H}$ statistic which provides a measure of the amount of uncertainty present in subjects' categories (Attneave, 1959; Garner, 1962). The consistency with which a subject classified a given stimulus is equivalent to $1-\mathrm{RelH}$, where RelH $=\Sigma$ plogp/\# of bits. Consistency scores for each of eight stimuli were computed separately for each subject. These scores were then submitted to an ANOVA of a 3 (condition) by 8 (stimulus) mixed design. Neither of the main effects (condition, $F(2,12)$ $=1.55$; stimulus, $F(7,84)=1.04$ ] nor the interaction $[F(14,18)=1.22]$ was significant, indicating that there was no difference in the consistency with which subjects sorted the syllables or either type of chirp pattern. More importantly, the overall consistency score of .78 (where minimum and maximum possible scores are 0.0 and 1.0 , respectively) demonstrates that subjects were able to reliably classify the stimuli into categories.

The kinds of groups that subjects formed depended somewhat on the stimulus condition. Each of the five subjects in the syllable condition sorted the eight sounds into four groups based on vowel identity: (bi, di), (be, de), (bo, do), (bə;, də). There was no indication of any other pattern prevalent in subjects' sorting of the syllables. Even the subject whose sorting fit the vowel-identity rule least well was accurate by this rule on $82 \%$ of the trials.

In contrast, no particular classification pattern predominated in the full-formant condition. However, individual subjects were all internally consistent in following the idiosyncratic classification schemes they had chosen. One subject actually followed the same pattern as those who sorted the syllables, but the remaining subjects grouped the stimuli in very different ways. Similarly, for the two-formant chirps, there was no particular pattern extant in the sorting behavior of all five subjects. Instead, a variety of patterns occurred. Interestingly enough, none of the subjects followed the vowel-identity rule with these stimuli, although there was some indication, present strongly in the data from one of the subjects, of division according to what might be termed "vowel similarity." On $100 \%$ of the trials, this subject sorted the chirps into two groups-(bi, be, di, $\mathrm{d} \varepsilon$ ) and (bar, bo, do, do)-which conforms to a split based upon a front-back vowel distinction. Thus, in no condition did the subjects group the sounds by categories corresponding to the initial consonant-although information about the nature of the initial consonant sounds was almost certainly available to subjects in the syllable condition.
The salience of the vowels in the syllable condition is perhaps not so surprising. After all, the vocalic portion of the syllables was much longer in duration than the consonantal portion. Interestingly, there is some indication that the full-formant chirps also preserve vowel information. One of the subjects in the full-formant condition employed a vowel-identity rule in grouping the sounds. This finding is consistent with those of Blumstein and Stevens (1980), who employed comparable stimuli and found that subjects were quite successful in labeling vowels. The picture for the two-formant patterns is less clear. No subject in this condition divided the stimuli into groups based upon vowel identity, although several subjects appeared to be following some sort of strategy based on vowel similarity. Thus, it is possible that both the full syllables and the brief beginning segments of those syllables contain both consonant and vowel information. This possibility was pursued in the second experiment by requiring subjects to sort the sounds into only two categories.

\section{EXPERIMENT 2}

In the previous study, no subject sorted the stimuli into groups based on the identity of the initial consonant sounds, even in the syllable condition. Instead, the most prevalent grouping was a division according to vowel identity. One factor which may have contributed to the choice of this classification scheme was the fact that subjects were not restricted as to the number of categories they could use in sorting the stimuli. The lack of such restriction plus the apparent salience of the vocalic portions of the stimuli may have encouraged subjects to group the stimuli into four equal-sized categories based on vowel identity.

As noted earlier, a given set of stimuli can be divided in a number of different ways. Just which way a subject chooses depends on a number of things, including the number of categories to be employed (Garner, 1974). The most obvious common factor for an unconstrained classification may not be the most obvious one for a division into two categories. Given the present set of stimuli, one might hypothesize that a classification according to the identity of the initial consonant would be enhanced if subjects were constrained to divide the stimuli into only two classes. Hence, in the present experiment, subjects were once again required to sort syllables, fullformant chirps, or two-formant chirps into groups, but this time, they were instructed to use only two groups.

\footnotetext{
Method

Stimuli. The stimulus materials were identical to those used in Experiment 1.

Subjects. The subjects were $\mathbf{3 0}$ undergraduates at Dalhousie University who received course credit for participating in the ex-
} 
periment. All were native speakers of English and reported no history of either speech or hearing disorder.

Procedure. The details of the procedure were virtually identical to those of Experiment 1. The only change introduced was that subjects were instructed to divide the stimuli into two classes. There were no other restrictions on the kinds of classes that subjects could form (e.g., the classes did not necessarily have to contain an equal number of members).

\section{Results}

Once again, only the data for the second 80 -item test block were analyzed. Consistency scores for each subject were computed using the RelH measure described in Experiment 1. The average consistency score across all conditions was .79 . The scores were submitted to an ANOVA of a 3 (condition) by 8 (stimulus) mixed design. In contrast to the previous experiment, there was a reliable main effect of stimulus $[F(7,189)=4.08, p<.05]$ and a marginal main effect of condition $[\mathrm{F}(2,27)=3.21]$. The interaction between these two factors $[F(14,189)<1.00]$ did not attain significance. Post hoc analysis conducted with Newman-Keuls tests $(p<.05)$ indicated that subjects were more consistent in classifying the syllables than the full-formant chirps, suggesting that the syllables were easier to remember. In addition, the consistency scores attained for [bi] stimuli (.91 overall) were reliably better than those for either $[b \varepsilon]$ or $\left[d^{*}\right](.68){ }^{4}$

For purposes of describing the classification used by individual subjects, each subject's data were scored according to the proportion of total trials which followed a particular sorting rule. In order to be classified as having used a particular sorting rule, a subject had to correctly sort the stimuli according to the rule on at least $80 \%$ of the trials. By this criterion, there were two prevalent patterns observed for the full syllables. Three of the subjects divided the syllables into two groups based on the identity of the initial consonant sounds-that is, ([bi], [be], [bət, [bo]) vs. ([di], [d $\varepsilon],\left[\mathrm{d} \jmath^{\star}\right]$, [do])-while five chose groups based on vowel similarity - that is, ([bi], [be], [di], [de]) vs. ([bo], [bə*], [do], [də]]). The grouping patterns followed by the remaining two subjects appeared to be idiosyncratic. With respect to the full-formant chirps, one subject sorted according to consonant identity and two subjects by vowel similarity. The sorting pattern of two of the remaining seven subjects was notable in that the stimuli were divided as follows: ([bi], [di]) vs. (all others). This classification scheme pits the two stimuli having the highest second- and third-formant offset values against all the other stimuli. The remaining five subjects in the full-formant chirp condition employed idiosyncratic sorting patterns. In contrast to the other two conditions, the data for the two formant chirp conditions are easily described, since all 10 subjects sorted according to the vowel similarity rule-i.e., ([bi], [bz], [di], [dE]) vs. ([bo], [bə^], [do], [də]).

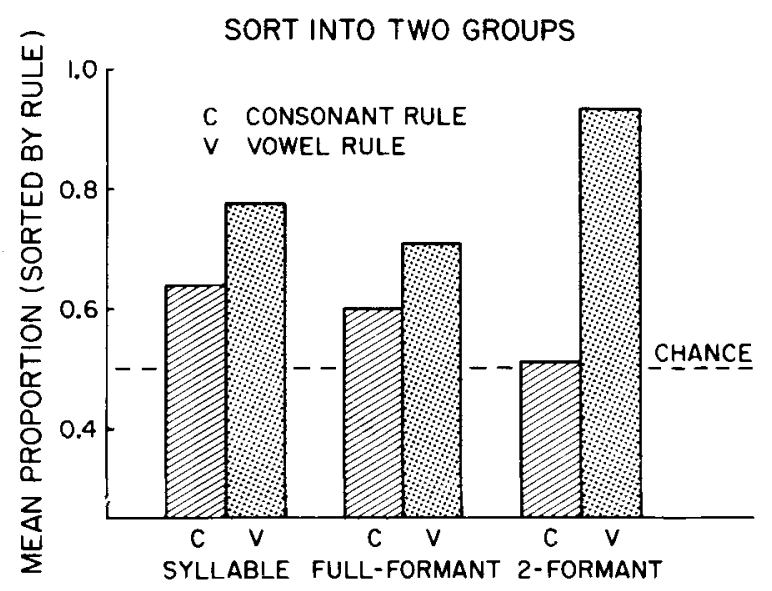

Figure 4. The mean proportion of trials correctly sorted by the consonant-identity and vowel-similarity rules for each of the three types of stimuli when subjects were instructed to sort the stimuli into two groups.

The predominant sorting patterns-vowel similarity and consonant identity - that emerged in the examination of the individual subject data were confirmed in an analysis of the group results. Figure 4 displays the mean proportion of trials correctly sorted by the vowel and consonant rules for each of the three conditions. It is readily apparent that across all three conditions, the preferred grouping was a division by vowel similarity. In each condition, sorting by the vowel similarity rule was reliably better than chance $(\mathrm{p}<.05$ or better by a $t$ test $)$. However, the degree to which the vowel similarity rule was applied differed across conditions, as the results of a oneway ANOVA revealed. There was a significant main effect of condition $[F(2,27)=6.02, p<.05]$, which a Newman-Keuls test indicated could be traced to greater use of this rule in the two-formant condition than in either of the other two conditions.

The consonant-identity rule was used much less widely by subjects. Only in the syllable condition did its usage reliably exceed that expected by chance.

\section{Discussion}

There are several points to be made about the results of this experiment. First, there is some evidence that the restriction to sort the stimuli into two categories increased the likelihood that subjects would form perceptual categories based on the identity of the initial consonants. More importantly, one of the four subjects who employed the consonant-identity rule did so with the full-formant chirp stimuli. This result alone suggests that there is usable information concerning the identity of the initial consonant present in the full-formant chirps. In contrast, there was no evidence that any of the subjects in the two-formant chirp condition utilized the consonant identity rule. 
Although some subjects grouped the stimuli on the basis of consonant identity, the majority of subjects appeared to follow a different sorting rule-based on vowel similarity. This grouping scheme, which was manifested in the behavior of a few subjects in Experiment 1 , was the only rule employed by subjects in the two-formant chirp condition. Moreover, half of the subjects in the syllable condition also appeared to follow this strategy.

What underlies this sorting by vowel similarity in the chirp conditions when only the formant transitions of the syllables are present? One clue comes from previous investigations in which similarity judgments have been collected for brief, rapidly changing acoustic segments. Brady, House, and Stevens (1961) required subjects to match the frequency of a steady tone to that of a short stimulus which underwent a rapid shift in frequency. They observed that there was a strong tendency for subjects to choose a frequency value which nearly matched the offset value of the rapidly changing stimuli. Similarly, in an experiment involving formant transition patterns not unlike the two-formant chirps in the present study, Shattuck and Klatt (1976) found that subjects favored matches between stimuli which shared offset values in the region of the second formant. Consequently, the results of these two studies suggest that the most likely grouping strategy for subjects in the present study would be to put together those stimuli most similar in their offset values. A classification according to vowel similarity would be expected in the present experiment if the categories were based upon the offset values of the formants, especially those of the second formant.

\section{EXPERIMENT 3}

The previous experiment suggests that there may be invariant cues for the identity of initial consonants available to the perceiver in the full-formant chirps. Yet, the use of these cues was not widespread, even among subjects who classified syllables. The chief factor here seems to be the greater salience of the offset values of the formants as a standard for classifying the stimuli into natural categories. One possible way of countering the predominance of the offset value cue would be to direct subjects to sort the stimuli according to their "initial sounds."

\section{Method}

Stimuli. The stimulus materials were identical in all respects to those used in the previous two experiments.

Subjects. The subjects were 15 undergraduates at Dalhousie University who received course credit for participating in the experiment. All subjects were native speakers of English and reported no history of either speech or hearing disorder.

Procedure. The procedure was virtually identical to that of Experiment 2, with the exception that subjects were instructed to sort the stimuli into two groups on the basis of their initial sounds.

\section{Results}

Only the data from the second 80 -item test block were analyzed. Again, consistency scores were computed for each subject using the RelH measure. An ANOVA of a 3 (condition) by 8 (stimulus) mixed design was used to analyze the consistency data. Neither of the main effects [condition, $F(2,12)=2.08$; stimulus, $F(7,84)=1.59$ ] was reliable, nor was the interaction between these factors $[F(14,18)<1.00]$. The average consistency score across all conditions was .82 .

An examination of the classifications imposed by individual subjects was conducted using the criterion of $80 \%$ correct sorts by a particular rule, as in Experiment 2. In the syllable condition, all five subjects grouped the stimuli by the consonant identity rule. In the full-formant condition, one subject grouped the stimuli by consonant identity, two by vowel similarity, and two by a grouping that pitted the [bi] and [di] stimuli against all others. Thus, although there was further confirmation that the full-formant chirps could be partitioned according to consonant identity, the change in instruction set did not appear to greatly lessen the tendency of most subjects to group according to the offset values of the stimuli. Finally, in the two-formant condition, four of the five subjects grouped according to the vowel-similarity rule. ${ }^{5}$ Once again, no subject in this condition gave evidence of using the consonant-identity rule.

Figure 5 displays the mean proportion of trials correctly sorted by the vowel and consonant rules in each condition. The most notable change between the patterns displayed here and those of the previous experiment occurs in the syllable condition. Usage of the vowel-similarity rule dropped to chance level, whereas usage of the consonant-identity rule increased to a level of $99 \%$ accuracy. Yet, although

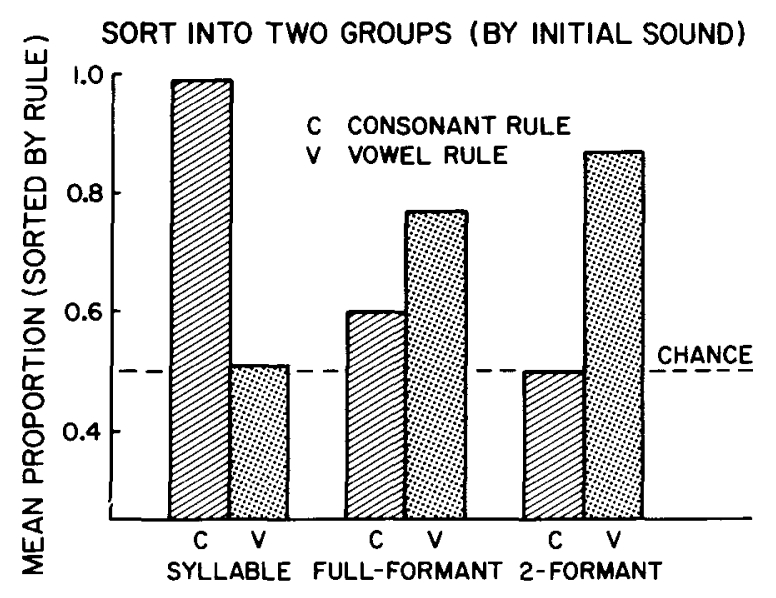

Figure 5. The mean proportion of trials correctly sorted by the consonant-identity and vowel-similarity rules for each of the three types of stimuli when subjects were instructed to sort the stimuli into two groups based on the identity of the initial sounds. 
the instruction set obviously altered the predominant model of classifying in the syllable condition, it did not appear to affect either of the two chirp conditions. In both of these conditions, sorting according to a vowel-similarity rule was reliably better than chance ( $p<.05$ or better by a t test), whereas sorting by the consonant-identity rule was not.

Further tests of the degree to which each sorting rule was used by subjects in each condition were conducted using one-way ANOVAs. For the consonantidentity rule there was a reliable main effect of condition $[F(2,12)=45.06, p<.001]$, which NewmanKeuls tests indicated could be traced to decreased usage of the rule from syllables to full-formant chirps to two-formant chirps. A similar analysis conducted on the vowel-similarity rule also found a main effect of condition $[F(2,12)=8.52]$ attributable solely to the infrequent usage of this rule in the syllable condition.

\section{Discussion}

The change in instruction set appears to have had its greatest impact on the performance of subjects in the syllable condition. All five subjects in this condition formed classes consistent with a division by initial consonant sound. In contrast, there was no observable change in the sorting patterns chosen by subjects in either of the two chirp conditions. Once again, similarities in the offset values of the stimuli were the dominant factor in the organization of stimulus groups, although, as in the previous experiment, one subject in the full-formant condition did follow a consonant-identity pattern. Thus, the change in instruction set was not sufficient to increase the use of the consonant-identity strategy in either of the chirp conditions.

To this point, then, there is little evidence that information is available in full-formant chirps that allows subjects to sort these stimuli into groups corresponding to the identity of initial consonants and no indication that any such information is available in the two-formant chirps.

\section{EXPERIMENT 4}

Although there is not a strong tendency for subjects to spontaneously group the chirps according to consonant identity, they still might be able to abstract the consonant information from these stimuli if required to do so. One way of requiring subjects to use information about consonant identity is to employ a perceptual learning task in which subjects are instructed to categorize sounds according to this rule. Grunke and Pisoni (Note 3 ) have shown that such a task may provide a more sensitive measure of the perceptual relatedness of auditory patterns than a similarity judgment task. To the extent that there is information available to the perceiver for a partition- ing of the stimuli by consonant identity, subjects should be able to learn to sort by this rule. More importantly, if the information in the chirps concerning consonant identity has any psychologically special status for the perceiver, it should be easier to learn to sort by this rule than by one that imposes an arbitrary organization on the stimuli.

The present experiment was undertaken to determine the degree to which subjects might be able to learn to classify the chirps according to a consonant identity rule. There were two components to this experiment. The first of these was a learning task in which each subject was required to classify a given set of sounds according to three different rules: consonantidentity, vowel-similarity, and arbitrary. ${ }^{6}$ The second phase of the present experiment was a speeded classification task in which subjects had to assign the stimuli to groups according to one of the three rules as rapidly as possible. Differences in the speed with which subjects were able to apply either the consonantidentity or the vowel-similarity rules relative to the arbitrary rule would provide a further index of the psychological status of these rules.

\section{Method}

Stimuli. The stimuli were identical to those used in the previous experiments. However, new types of demonstration and training tapes were employed. The demonstration tapes were constructed so as to correspond to one of the three rules. For example, the demonstration tape for the consonant-identity grouping of the syllables consisted of all the members of one category (e.g., [bi], [be], [bo], [ba^]) presented consecutively at $1-\mathrm{sec}$ intervals. After a short pause, the members of the other category were presented in the same manner. Following another pause, the category 1 items recurred but in a different order, followed again by the category 2 items, also in a different order. Demonstration tapes for each stimulus type (syllable, full-formant chirp, two-formant chirp) by grouping rule (consonant-identity, vowel-similarity, arbitrary) combinations were prepared in a similar fashion.

The training tapes employed in this experiment consisted of randomized sequences of the eight stimuli in a given stimulus condition. There were a total of three occurrences of each syllable for a total of 24 trials per tape spaced at 4-sec intervals. Three separate training tapes with different random orders were prepared for each stimulus condition.

The nine test tapes were prepared in the same manner as those employed in the previous experiments.

Subjects. The subjects were 18 undergraduates at Dalhousie University who received course credit for their participation in the experiment. All were native speakers of English and reported no history of either speech or hearing disorders.

Procedure. Each subject was assigned randomly to one of the three stimulus conditions (i.e., full-CV syllables, full-formant chirps, or two-formant chirps). Within a given stimulus condition, the subjects were trained to sort the stimuli according to each of three sorting rules. These rules are displayed in Table 2 . Thus, the consonant-identity rule required subjects to group together stimuli that shared the same initial consonant (i.e., [b] vs. [d]). The vowel-similarity rule required subjects to group stimuli from syllables containing the [i] and $[\varepsilon]$ vowels into one category and the $[0]$ and $\left[\partial^{\circ}\right]$ vowels into another. Finally, the arbitrary rule grouped together stimuli which shared neither a consistent consonant identity nor a consistent vowel similarity. The order of learning the three rules was counterbalanced within each condition. Training 
Table 2

Three Sorting Rules for Learning and Speeded Classification Tasks

\begin{tabular}{|c|c|c|c|c|c|}
\hline \multicolumn{2}{|c|}{ CONSONANT } & \multicolumn{2}{|c|}{ VOWEL SIMILARITY } & \multicolumn{2}{|c|}{ ARBITRARY } \\
\hline$[\mathrm{bi}]$ & {$[\mathrm{di}]$} & [bi] & [bo] & [bi] & [di] \\
\hline \multicolumn{2}{|l|}{ [De } & [di] & [do] & {$[b o]$} & {$[d o]$} \\
\hline [bo] & {$[\mathrm{do}]$} & {$[b \epsilon]$} & {$[b \sigma]$} & {$[d \epsilon]$} & {$[b \epsilon]$} \\
\hline [bor] & {$\left[d{ }^{\prime}\right]$} & {$[\mathrm{d} \epsilon]$} & {$[d r]$} & {$[d r]$} & [bø'] \\
\hline
\end{tabular}

took place separately for each rule and proceeded in the following manner. A subject was informed that he or she would be hearing eight different sounds and that four of these were to be assigned to category 1 and four to category 2 . The experimenter then played the four stimuli corresponding to category 1, stopped the recorder, and informed the subject that the next four items were members of category 2 . After the category 2 members had been presented, the tape was stopped again, at which point the experimenter repeated the instructions and demonstrated the categories for the subject a second time. The subject was then informed that he or she would receive a set of training trials during which each stimulus was to be assigned to either category 1 or category 2 , and that feedback as to the correctness of a response would be provided on each trial. The assignment of a given stimulus to a particular category was made by pressing buttons labeled 1 and 2 located on a box situated next to the subject's right hand. The subject was instructed to place the right index finger on one button and the right middle finger on the other. For a given subject, the location of the category 1 and category 2 buttons remained constant throughout the experiment. However, the ordering of the buttons was counterbalanced across subjects.

A subject was deemed as having successfully learned a given rule if he or she answered correctly on 20 of the 24 training trials in a block. If, at the end of the first block of training trials, the subject had not met the learning criterion, the demonstration tapes were played once again for the subject and a second block of training trials was run. Testing in this manner until either a subject successfully learned the rule or a total of four training blocks were completed without the subject's having learned the rule. ${ }^{7}$ In the latter circumstance, testing on the rule was terminated and the subject began training on any other rule that remained to be learned.

If a subject had successfully learned a particular rule, then he or she was immediately tested on the corresponding speeded classification task prior to learning another classification rule. The instructions for the speeded classification task were essentially the same as for the learning task. However, the subjects were told to respond as rapidly as possible without making errors. Moreover, no feedback was provided regarding the correctness of responses. An 80-item test block (10 occurrences of each of the eight stimuli), consisting of a random arrangement of the stimuli spaced at 4-sec intervals, was then presented to the subject. On each trial, a digital timer was activated by the first voicing pulse from the stimulus. The timer was halted as soon as the subject pressed one of the two response keys or if the subject failed to respond within $3 \mathrm{sec}$ (at which point a reaction time of $3,000 \mathrm{msec}$ was recorded). On each trial, the experimenter recorded both the subject's response and the reaction time. Two small lights indicated to the experimenter which response key had been pressed on a given trial. Both the response lights and the timer, though visible to the experimenter, were out of view for the subject. Upon completion of the 80th trial, a 5-min break period ensued, after which training began on a new classification rule. Training and testing proceeded as before until a subject had completed training on all three rules. An entire experimental session took approximately $1 \mathrm{~h}$.

\section{Results}

The learning data were examined in two ways. First, the number of subjects who successfully completed training on a given rule in a particular condition was calculated. In the syllable condition, all subjects passed the training phase for each rule; the situation was considerably different for the two chirp conditions. In the full-formant condition, all subjects passed the training phase for both the vowel-similarity and consonant-identity rules, but only one subject successfully completed training with the arbitrary rule. In the two- formant condition, all subjects successfully mastered the vowel similarity rule, but not a single subject passed the training criterion with either the consonant-identity or arbitrary rules. These results are quite informative because they indicate that although subjects can learn to classify the fullformant chirps on the basis of consonant-identity, they are unable, within the learning criterion, to classify two-formant chirps on this basis.

The number of errors made during the training tasks was also analyzed. The mean number of errors made in learning each rule is displayed for the three stimulus conditions in Figure 6. The error data for individual subjects were submitted to an ANOVA of a 3 (condition) by 3 (classification rule) mixed design. Both main effects [condition, $F(2,15)=112.98, p<.01$;

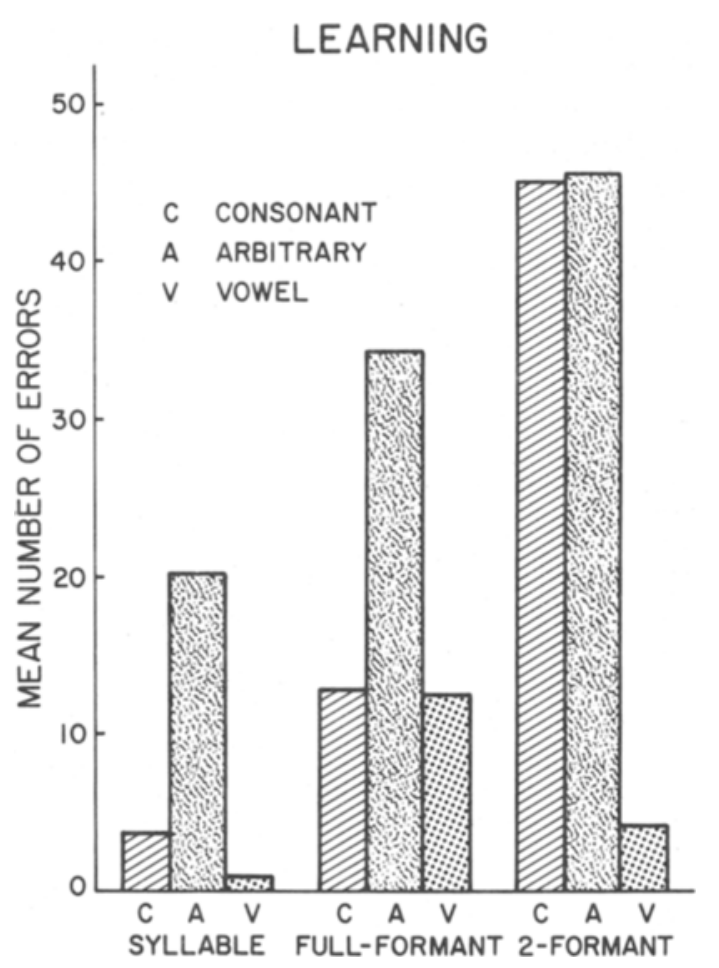

Figure 6. Mean number of errors for each type of stimulus when subjects were forced to sort the stimuli according to the consonant-identity, arbitrary, and vowel-similarity rules. 
classification, $F(2,30)=73.99, \mathrm{p}<.01]$ and the interaction $[F(4,30)=21.40, p<.01]$ were significant. Newman-Keuls tests with a criterion level of $p<.05$ were used to conduct a post hoc analysis of the results. The critical comparison in each case is how performance with the consonant-identity or vowelsimilarity rule differs from that with the arbitrary rule. In both the syllable and full-formant conditions, significantly more errors were made in learning the arbitrary rule than in learning either the consonantidentity or vowel-similarity rules, which did not differ from each other. Hence, both rules appear to have a psychologically special status for subjects in the syllable and full-formant conditions. This was not the case for the two-formant condition. Only errors in learning the vowel-similarity rule were reliably less than errors in learning the arbitrary rule. Thus, it appears that if there are any invariant cues to consonant identity in the two-formant chirps, they may be inaccessible to the perceiver. That is, the psychological status of any potential invariants in the twoformant chirps is questionable.

An indication of the ease with which a given rule was learned across the three stimulus conditions was obtained from additional post hoc tests on the error data. With the consonant-identity rule, there were no reliable differences between the syllable and fullformant conditions, though both were obviously superior to the two-formant condition. With the vowel-similarity rule, there were no reliable differences between any of the conditions. Finally, with the arbitrary rule, there were considerably fewer errors in the syllable condition than in either of the other two.

The speeded classification data, displayed in Table 3, exhibit much the same pattern as the learning data. The reaction time data reinforce the conclusion that there is psychologically usable information regarding consonant identity available in both the syllables and full-formant chirps. A one-way ANOVA conducted on the syllable condition indicated a reliable main effect $[F(2,12)=9.82, p<.01]$ traceable to the fact that reaction times were significantly slower with the arbitrary rule. The pattern of no reliable differences in reaction time for the consonantidentity and vowel-similarity rules present in the syl-

Table 3

Speeded Classification Experiment

\begin{tabular}{c|c|c|c}
\hline & \multicolumn{3}{|c}{ RULE USED TO SORT STIMULI } \\
& $\begin{array}{c}\text { VOWEL } \\
(n=6)\end{array}$ & $\begin{array}{c}\text { CONSONANT } \\
(n=6)\end{array}$ & $\begin{array}{c}\text { ARBITRARY } \\
(n=5)\end{array}$ \\
\hline $\begin{array}{c}\text { SYLLABLES } \\
\text { FULL-FORMANT } \\
\text { PATTERNS }\end{array}$ & 787 & 857 & 1057 \\
$\begin{array}{c}\text { 2-FORMANT } \\
\text { PATTERNS }\end{array}$ & 905 & 843 & - \\
\hline
\end{tabular}

Note-Median reaction times in milliseconds. lable condition also held for the full-formant condition $[\mathrm{t}(5)<1.00]$. Finally, a comparison of the reaction time data across stimulus conditions was not conducted because the different lengths of the syllables and chirps rendered such a comparison uninterpretable.

\section{Discussion}

The present results provide very clear evidence that the full-formant chirps contain sufficient information, in a form accessible to the perceiver, to allow them to be grouped according to consonant-identity. That this information has a psychologically special status for the perceiver is shown by the fact that a grouping by consonant identity was much more readily learned than was one by an arbitrary rule. This performance difference between the consonant identity and arbitrary rules is crucial, since it serves to establish that the use of the consonant-identity rule involved more than mere rote memorization of the stimulus sets. At the same time, there is every indication that the two-formant chirps do not contain sufficient acoustic information to allow for sorting on the basis of consonant identity.

But the information specifying consonant identity is not the only psychologically usable information in the stimuli, as the results for the vowel-similarity rule demonstrate. Across all stimulus conditions, subjects' mastery of this rule was clearly superior to their mastery of the arbitrary one. Not only was this rule easier for subjects to learn, but also, in the one condition in which subjects did learn the arbitrary rule (i.e., syllable condition), speeded sorting was reliably faster with the vowel-similarity rule than with the arbitrary rule. Thus, the present results confirm the observation made on the basis of the earlier experiments that there is information in the stimuli which leads to a division of the sounds by vowel similarity.

One additional point needs to be made about the present experiment. The perceptual learning task proved to be a more sensitive measure of whether subjects could access information that would allow them to sort by a consonant-identity rule. It seems likely that a task of this type is apt to be most useful in those situations in which there is potentially a very powerful perceptual classification scheme which may overwhelm alternative ways of organizing the stimulus sets. In such circumstances, a perceptual learning task, wherein performance under a given classification scheme is compared with that under an arbitrary scheme, may prove to be the most appropriate means of establishing the psychological status of the classification scheme and the stimulus dimensions.

\section{GENERAL DISCUSSION}

The main objective of the present investigation was to determine whether brief portions of the speech signal present at the onset of $\mathrm{CV}$ syllables contained 
sufficient information for listeners to categorize utterances of the same consonant occurring in different vowel contexts. The results, especially those of Experiment 4 , demonstrate convincingly that such information is available to the listener in the onsets of brief segments of speech containing five formants. Hence, the present investigation nicely complements those which have employed new analyses of the acoustic signal (e.g., Kewley-Port, 1980; Searle et al., 1979; Stevens \& Blumstein, 1978). These new analyses suggest that there is physically invariant information in the first $30 \mathrm{msec}$ of speech, and the present results show that there is psychologically usable information within this period.

However, neither the present results nor those of Blumstein and Stevens (1980) indicate the physical basis by which subjects categorize the sounds. As such, the present results do not distinguish which, if any, of the alternative descriptions of invariant properties in the physical signal best correspond to the one used by the perceiver. Thus, accounts that derive physical invariants from computations over running spectra (e.g., Searle et al., 1979; Kewley-Port, Note 2) are just as consistent with the present results as are those based on averaged onset spectra (e.g., Stevens \& Blumstein, 1978, in press) provided they locate the source of the invariance within the first $30 \mathrm{msec}$ of the signal.

Although there is information available in the fullCV syllables and full-formant chirps that allows them to be grouped by consonant identity, it is clear that such a grouping is not the one most preferred by subjects. Throughout this series of experiments, and with all stimulus sets, subjects appeared to favor classifications that grouped together stimuli having similar offset values. This grouping was designated "vowel-similarity," since it divided the stimuli in a way which corresponded to a front-back vowel distinction. The finding that subjects grouped the stimuli in terms of offset values is not without precedent. The work of Brady et al. (1961) and Shattuck and Klatt (1976) indicated that subjects tend to match brief, rapidly changing acoustic patterns in terms of their offset values; and this tendency is not limited solely to brief patterns, as Grunke and Pisoni (Note 3) discovered. Using patterns of durations similar to those in the present study, Grunke and Pisoni found that subjects most readily learned to match patterns having similar offset values. Moreover, they also discovered that adopting a procedure in which the differences in the offset values of the stimuli were reduced enabled subjects to match rather dissimilar rise-fall patterns as readily as mirror-image acoustic patterns. Hence, it is clear that offset frequency information is particularly salient for the listener. Indeed, in the present experiment, subjects whose groupings followed the vowel-similarity pattern reported on the questionnaire that pitch was the predominant factor in selecting their groups. Many described their classifications as "high pitch" vs. "low pitch." Thus, it may be more appropriate to describe these "vowel-similarity" classes as following a pitch similarity rule, especially since the vowel similarity rule appears to be a special subset of the former. ${ }^{8}$

Finally, the present results raise serious doubts about whether two-formant chirps lacking firstformant cues are suitable for use as nonspeech controls in speech perception experiments. In the past, chirps lacking first-formant information have often been used as control stimuli because it was claimed that they presented the listener with the same critical information (i.e., acoustic cues) contained in syllables, but in a nonspeech context (e.g., Mattingly et al., 1971). First-formant information was routinely deleted from such chirps to make them less speech-like. The justification offered for this procedure was that the first-formant information did not help to distinguish the speech contrasts under investigation-for example, [b] and [d]. However, this argument holds only under the assumption that the information content of each formant is independent of the other formants. If one assumes, instead, that it is the relationship that exists between the formants which is critical for perception, then the first formant information cannot be considered to be redundant. The present results support the latter view because they show that, whereas the two formant chirps do not contain the information necessary to permit a grouping according to consonant identity, such a grouping is easily achieved with patterns that do preserve first-formant information-viz., the fullformant chirps. A similar view regarding the importance of first-formant information for the perception of brief auditory patterns has been expressed by Pisoni (Note 4). He observed that the inclusion of first-formant information in chirp patterns resulted in sharp discontinuities in discrimination functions in the vicinity of phonetic boundaries. Such discontinuities are not typically present for patterns lacking first formant information (e.g., Mattingly et al., 1971). Hence, it seems clear that acoustic energy in the region of the first formant plays a critical role in the perception of the overall acoustic pattern.

In summary, the present results suggest that there is psychologically usable information available in the onsets of syllables which may allow the listener to perceive the same consonant in different vowel contexts. Such findings suggest that previous studies may have underestimated the degree of invariance present in the perceptual structure of speech stimuli.

\section{REFERENCE NOTES}

1. Kewley-Port, D. KLTEXC: Executive program to implement the KLATT software synthesizer. Research on Speech Perception, 1978, Progress Report 4, Indiana University. 
2. Kewley-Port, D. SPECTRUM: A program for analyzing the spectral properties of speech. Research on Speech Perception, 1979, Progress Report No. 5, Indiana University.

3. Grunke, M. E., \& Pisoni, D. B. Some experiments on perceptual learning of mirror-image acoustic patterns. Paper presented at the Ninth International Congress of Phonetic Sciences, Copenhagen, 1979.

4. Pisoni, D. B. Discrimination of brief frequency glissandos. Research on Speech Perception, 1976, Progress Report No. 3, Indiana University.

5. English, R., Jusczyk, P. W., \& Smith, L. B. Factors affecting the perceptual classification of speech. Manuscript in preparation, 1981.

\section{REFERENCES}

AtTne Ave, F. Applications of information theory to psychology. New York: Holt, Rinehart \& Winston, 1959.

Blumstein, S. E., \& Stevens, K. N. Acoustic invariance in speech production: Evidence from measurements of the spectral characteristics of stop consonants. Journal of the Acoustical Society of America, 1979, 66, 1001-1017.

Blumstein, S. E., \& Stevens, K. N. Perceptual invariance and onset spectra for stop consonants in different vowel environments. Journal of the Acoustical Society of America, 1980, 67, 648-662.

Boning, E. G. Auditory theory with special reference to intensity, volume, and localization. American Journal of Psychology, 1926, 37, 157-188.

Boring, E. G. The physical dimensions of consciousness. New York: Century, 1933.

Brady, P. T., House, A. S., \& Stevens, K. N. Perception of sounds characterized by rapidly changing resonant frequency. Journal of the Acoustical Society of America, 1961, 33, 13571362.

Cole, R. A., \& Scotr, B. The phantom in the phoneme: Invariant cues for stop consonants. Perception \& Psychophysics, 1974, 15, 101-107.

Dondens, F. C. Über die Natur der Vocale. Beiträge zur Natur und Heilkundes, 1857, 1, 157-162.

Dorman, M., Studdert-Kennedy, M., \& Raphael, L. J. Stop consonant recognition: Release bursts and formant transitions as functionally equivalent context-dependent cues. Perception \& Psychophysics, 1977, 22, 109-122.

Ermas, P. D. Auditory and phonetic coding of the cues for speech: Discrimination of the [r-1] distinction by young infants. Perception \& Psychophysics, 1975, 18, 341-347.

FAnT, G. Acoustic theory of speech production. The Hague: Mouton, 1960.

GARNER, W. R. Uncertainty and structure as psychological concepts. New York: Wiley, 1962.

GaRner, W. R. The processing of information and structure. Potomac, Md: Erlbaum, 1974.

GiBson, J. J. The senses considered as perceptual systems. Boston: Houghton Mifflin, 1966.

Harris, K. S. Cues for the discrimination of American English fricatives in spoken syllables. Language and Speech, 1958, 1, 1-7.

HelmholTz, H. Sensations of tone (trans. A. J. Ellis; 5th ed.). New York: Longmans, Green, 1930.

Kempel.eN, W. Le mécanisme de la parole suivi de la description d'une machine parlante. Vienna: Imprimé chez $\mathrm{B}$. Baurr et se trouvé chez J. V. Degen, 1791.

KEWLEY-Port, D. Representations of spectral change as cues to place of articulation in stop consonants. Unpublished doctoral dissertation, City University of New York, 1980.

KIATT, D. H. Software for a cascade/parallel formant synthesizer. Journal of the Acoustical Society of America, 1980, 67, 971-995.

KoEnıG, R. Sur les notes fixes caractéristiques des diverse voyelles. Comptes Rendus Hebdomadaires des Séances der l'académie des sciences Paris, 1870, 70, 931-933.
KoHler, W. Akustiche Untersuchungen II. Zeitschrift für Psychologie, 1910, 58, 59-140.

Liberman, A. M., Cooper, F. S., Shankweiler, D. D., \& Studdert-Kennedy, M. Perception of the speech code. Psychological Review, 1967, 74, 431-461.

Liberman, A. M., Delattre, P. D., \& Cooper, F. S. The role of selected stimulus variables in the perception of the unvoiced stop consonants. American Journal of Psychology, 1952, 65, 497-516.

Mattingly, I. G., Liberman, A. M., Syrdal, A. K., \& Halwes, T. Discrimination in speech and nonspeech modes. Cognitive Psychology, 1971, 2, 131-157.

Miyawaki, K., Strange, W., Verbrugge, R., Liberman, A. M., Jenkins, J. J., \& Fujimura, $O$. An effect of linguistic experience: The discrimination of [r] and [l] by native speakers of Japanese and English. Perception \& Psychophysics, 1975, 18, 331-340.

Morse, P. A. The discrimination of speech and nonspeech stimuli in early infancy. Journal of Experimental Child Psychology, $1972,14,477-492$.

Schatz, C. D. The role of context in the perception of stops. Language, 1954, 30, 47-56.

Searle, C. L., Jacobson, J. Z., \& Rayment, S. G. Phoneme recognition based on human audition. Journal of the Acoustical Society of America, 1979, 65, 799-809.

Shattuck, S. R., \& KLATT, D. H. The perceptual similarity of mirror-image acoustic patterns in speech. Perception \& Psychophysics, 1976, 20, 470-474.

Smith, L. B., \& KemLer, D. G. Levels of experienced dimensionality in children and adults. Cognitive Psychology, 1978, 10, 502-532.

Stevens, K. N., \& Blumstein, S. E. Invariant cues for place of articulation in stop consonants. Journal of the Acoustical Society of America, 1978, 64, 1358-1368.

Stevens, K. N., \& Blumstein, S. E. The search for invariant acoustic correlates of phonetic features. In P. D. Eimas \& J. L. Miller (Eds.), Perspectives on the study of speech. Hillsdale, N.J: Erlbaum, in press.

\section{NOTES}

1. These findings were challenged some years later by Cole and Scott (1974), who argued that the burst information produced at the release of stop consonant occlusion serves as an invariant acoustic cue for the recognition of stop consonants in different vowel contexts. Subsequently, a more systematic investigation using a wider variety of vowel contexts and more precise controls of stimulus parameters indicated that the burst information by itself was not a sufficient cue for recognizing stops in all contexts (Dorman, Studdert-Kennedy, \& Raphael, 1977). Rather, bursts and formant transitions appear to be functionally equivalent, context-dependent cues which are reciprocally related such that when the perceptual weight of one increases, the weight of the other declines.

2. While initial burst information is typically included in the onset spectra of stop consonants, Blumstein and Stevens (1980) have noted that the onset spectra for stops generated without bursts do not differ appreciably. The main effect of including the burst is to enhance the global property which cues a particular place of articulation. As a check on the results of the present investigation, we have conducted a further series of experiments using stimuli containing burst information. The results of that study, which will be reported in a forthcoming paper (English, Jusczyk, \& Smith, Note 5) are substantially the same as those reported here.

3. We would like to thank Diane Kewley-Port for deriving the onset spectra for us.

4. It is difficult to know just what to attribute the difference in consistency scores to. While one possibility is that the $[\mathrm{b} \varepsilon]$ and $\left[\mathrm{d} \partial^{\wedge}\right]$ stimuli may have been more ambiguous than the [bi] stimuli, this pattern did not recur in either Experiment 1 or Experiment 3. Thus, it seems preferable to treat it as just an anomaly in the data. 
5. There is some indication that this subject became confused and reversed the numbers he assigned to each category midway through the test series. In fact, if the category assignments are simply reversed at a point midway through the series, the data follow a vowel-similarity pattern. It is also worth noting that this subject did sort the stimuli according to a vowel-similarity rule during the first 80-item test block.

6. The vowel-similarity rule was chosen since many subjects seemed to regard it as the most natural way of grouping the stimuli. Thus, it provided a convenient upper bound for measuring the ease with which the consonant-identity rule was learned.
7. Note that, since only three training tapes had been prepared for each stimulus condition, any subject who required four training blocks heard one of the training tapes twice. The choice of which tape was to be repeated was counterbalanced across subjects.

8. The notion that vowels might be classified with respect to pitch is a very old one in psychology, traceable back at least to Kempelen (1791) and also investigated by, for example, Donders (1857), Helmholtz (1930), Koenig (1870), and Kohler (1910).

(Manuscript received February 14, 1981; revision accepted for publication May 8, 1981.) 